\title{
More friend than foe: the emerging role of neutrophils in tissue repair
}

\author{
Moritz Peiseler ${ }^{1,2}$ and Paul Kubes ${ }^{1,2,3}$ \\ 'Department of Pharmacology and Physiology, ${ }^{2}$ Snyder Institute for Chronic Diseases, and ${ }^{3}$ Department of Microbiology, Immunology and Infectious Diseases, Cumming School of Medicine, \\ University of Calgary, Calgary, Alberta, Canada.
}

\begin{abstract}
Neutrophils are the most abundant immune cells in humans and serve as first responders to a myriad of host perturbations. Equipped with a plethora of antimicrobial molecules, neutrophils invade sites of inflammation to eradicate pathogens and clear debris. Traditionally, neutrophils were thought to cause collateral tissue damage before dying at the site. However, the presence of neutrophil infiltration into sterile injuries (in the absence of infections) suggests additional roles for these cells. Now, the view of neutrophils as indiscriminate killers seems to be changing as evolving evidence suggests that neutrophils actively orchestrate resolution of inflammation and contribute to tissue repair. Novel concepts include the idea that neutrophils are key to revascularization and subsequently reverse-transmigrate back to the vasculature, actively leaving sites of tissue damage to re-home to functional niches in the lung and bone marrow. This Review scrutinizes the role of neutrophils in tissue damage and repair, discussing recent findings and raising unresolved questions around this intriguing immune cell.
\end{abstract}

\section{Introduction}

Neutrophils are the most abundant immune cells, constituting about $60 \%$ to $70 \%$ of all leukocytes in human blood $(1,2)$. Neutrophils act as the first responders of the innate immune system, and their crucial role in fighting invading pathogens is well established and best exemplified by the severe susceptibility of neutropenic patients to infections $(3,4)$. The works of Paul Ehrlich in the late nineteenth century first recognized heterogeneity of leukocytes and identified one unique cell with a polymorphous nucleus as the "neutrophil" $(1,5)$. Neutrophil function was subsequently studied by Élie Metchnikoff, widely considered the father of cellular innate immunity, who first described recruitment of phagocytic cells to an injury in starfish embryos $(6,7)$.

However, until recently, the prevailing view of neutrophils was that of simple foot soldiers of the innate immune system: equipped with a lethal arsenal of proteases and oxidants, neutrophils rapidly invade sites of infection to eradicate pathogens and prevent their spread $(8,9)$. Upon completion of their tasks, neutrophils were thought to commit suicide on the battlefield. Overexuberant neutrophil recruitment was associated with collateral tissue damage, defective healing, and chronic inflammation (2). Adding to this was the discovery of NETosis (10), a novel killing mechanism by which neutrophils release neutrophil extracellular traps (NETs), nuclear DNA coated with histones, proteases, and granular and cytosolic proteins to entrap bacteria. While effective in capturing bacteria, NETs produced in infections and noninfectious perturbations have been postulated to cause bystander tissue damage (11).

The prevailing and rather simplistic view of the neutrophil has undergone substantial revision in the past decade, and numerous

Conflict of interest: The authors have declared that no conflict of interest exists. Copyright: (5) 2019, American Society for Clinical Investigation.

Reference information: / Clin Invest. 2019;129(7):2629-2639.

https://doi.org/10.1172/JCI124616. novel paradigms have emerged (12). Advanced techniques, such as intravital microscopy, genetic fate mapping, and single-cell sequencing, have driven considerable research in the field, spawning studies into more complex neutrophil biology. Furthermore, the identification of Ly6G as a lineage-specific neutrophil membrane protein that can be used to track or deplete neutrophils and the generation of the "Catchup mouse," a Ly6G neutrophilspecific, Cre-based reporter system driven by the Ly6G promoter combined with fluorescent tdTomato expression, have substantially advanced the study of neutrophils in vivo (13).

It is now apparent that neutrophils have crucial homeostatic functions in various organ systems $(14,15)$ : they interact with cells of the innate and adaptive immune system to direct immune responses (16), are implicated in chronic inflammatory diseases (17), experience shaping by the microbiome (18), and contribute to injury repair. Tumors may also hijack these properties to aid in growth and metastasis (19). Yet, despite encouraging advancements in many areas in recent years, some fundamentally unresolved questions remain (20). In this Review, we outline the neutrophil's role in tissue injury and repair, focusing on its emerging role in resolving inflammation and participation in repair. Since the mechanisms by which neutrophils are integrated in resolution are likely context-dependent, we also highlight neutrophil contributions to repair in different organs.

\section{Neutrophil recruitment}

Tissue injury leads to the release of an array of signals, including damage-associated molecular patterns (DAMPs) from damaged cells or pathogen-associated molecular patterns (PAMPs) in infection. Tissue-resident cells including macrophages, dendritic cells, and endothelium detect these signals, initiating neutrophil recruitment. As the first wave of infiltrating cells, neutrophils integrate these cues into a directed movement toward the injury site (21). Neutrophils express a multitude of receptors, including 


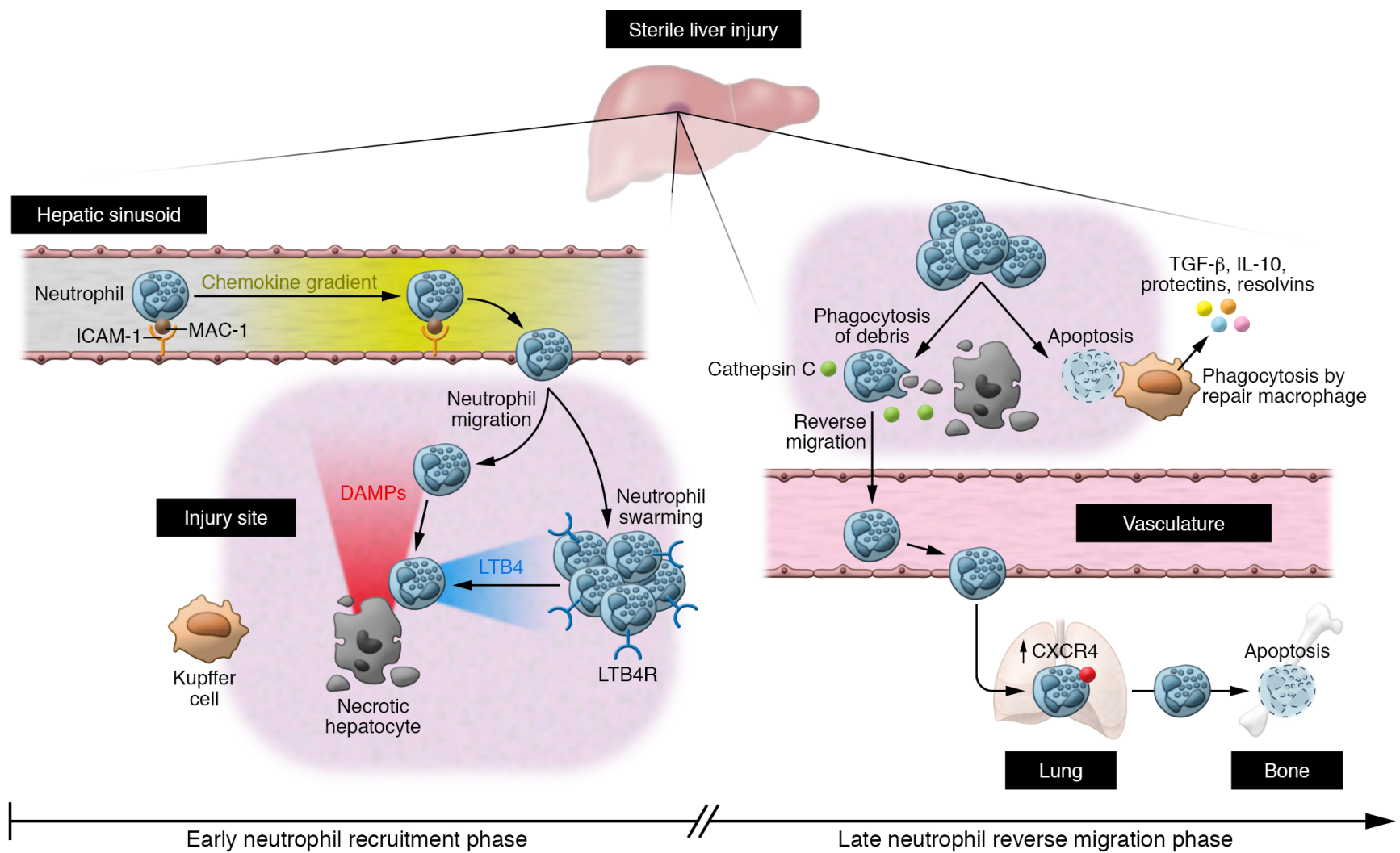

Figure 1. Spatiotemporal neutrophil response in focal necrotic liver injury. Left: Early neutrophil recruitment phase. Neutrophils are recruited as early as 15 to 30 minutes to the site of injury and fully cover the necrotic area within 4 hours. Their adhesion to hepatic sinusoids is achieved by binding of MAC-1 to endothelial ICAM-1. Recruitment occurs via CXC chemokine gradients along the endothelium, and closer to the focal necrosis, chemotaxis is guided by danger signals such as the $\mathrm{N}$-formyl peptides, ATP and HMGB1. In a second phase known as neutrophil swarming, additional neutrophils are recruited and leukotriene $B_{4}$ (LTB4) provides signal amplification. Tissue-resident macrophages called Kupffer cells serve as sentinels, detecting DAMPs and mediating recruitment of neutrophils. Right: Late neutrophil reverse migration phase. Over the next few hours, neutrophils phagocytose debris, dismantle vessels, and show a random back-and-forth movement. At the injury border, neutrophils show a directed movement away from the injury toward vessels, eventually clearing the injury site via the vasculature. CTSC is required for reverse migration by activating neutrophil serine proteases. Neutrophils migrate toward the lung vasculature, upregulating CXCR4 and eventually traveling to bone marrow, where they die by apoptosis. Some neutrophils die on site and are phagocytosed by resident or infiltrating macrophages, leading to macrophage phenotype switching and release of pro-resolving mediators. LTB4R, leukotriene $B_{4}$ receptor.

GPCRs, Fc receptors, adhesion receptors, cytokine receptors, and pattern recognition receptors, that allow recognition and response to injury and infection $(22,23)$. Neutrophil recruitment can be divided into an early phase induced by short-term signals and an amplification phase with robust neutrophil infiltration driven by more persistent signals (24). The general recruitment cascade of the neutrophil is an orchestrated process and has been extensively reviewed $(4,25,26)$; thus, we will limit our focus to recruitment during sterile injury.

DAMPs released from necrotic tissues directly recruit neutrophils (27). Neutrophil-derived ATP can augment chemotaxis in an autocrine fashion, providing signal amplification for neutrophil migration (28). Additional early signals are provided by $N$-formyl peptides such as fMet-Leu-Phe (fMLP), a key chemoattractant that is canonically thought to be bacteria-derived but is also released from damaged mitochondria during tissue necrosis. fMLP acts via formyl peptide receptors 1-3 (FPR1-3) (refs. 29, 30, and Figure 1). FPR1 deficiency in mice was shown to prevent neutrophil migration toward a focal hepatic injury (31), and a similar role in neutrophil migration was observed in humans (32). FPR2 mediates both pro- and antiinflammatory effects through binding of different chemotactic ligands (33). In atherosclerosis, binding of the resolving ligand annexin $\mathrm{A} 1$ (AnxA1) dampened neutrophil recruitment, leading to improved atherosclerotic lesions (34). Conversely, cathelicidin-related antimicrobial peptide (CRAMP), a proinflammatory FPR2 ligand, promoted atherosclerosis and was found to be deposited by neutrophils (35). Neutrophils produce leukotriene $\mathrm{B}_{4}$ (LTB4) in response to fMLP and sense LTB4 via the LTB4 receptor (24). Through this mechanism, neutrophils beget an abundance of additional neutrophils, an effect known as "neutrophil swarming" (36). LTB4 released from early-recruited neutrophils acts as a relay chemoattractant to mediate this phase of exponential neutrophil influx $(24,36)$. In a mouse model of skin injury, LTB4 was required for the recruitment of more distant neutrophils, while more proximal neutrophils in LTB4-deficient mice could still undergo chemotaxis to the injury site (37). The size of an injury could dictate how many neutrophils are recruited by the primary end-target signal (e.g., fMLP) and how many are recruited by relay chemoattractants 


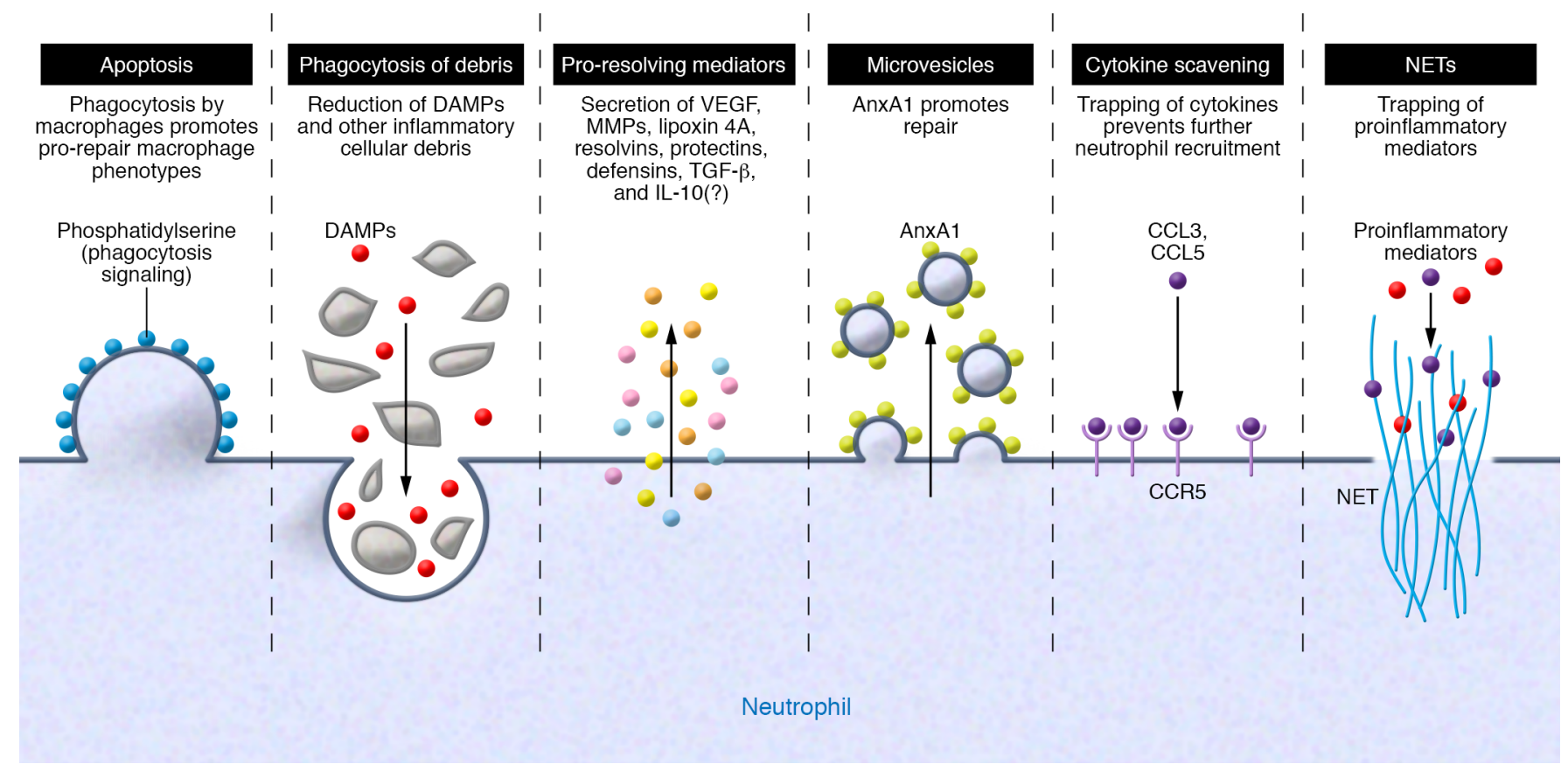

Figure 2. The pro-resolving/pro-repair neutrophil. Neutrophils use different strategies to initiate tissue repair, which often occur simultaneously or sequentially. From left to right: Neutrophils undergo apoptosis and expose "eat-me" signals such as phosphatidylserine on the cell surface, leading to phagocytosis by resident macrophages and inducing a pro-repair feed-forward loop. Neutrophils phagocytose debris, thereby clearing the injury site of proinflammatory stimuli, removing dead tissue, and making channels for angiogenesis. Neutrophils release numerous mediators that promote angiogenesis and tissue repair and modulate the inflammatory milieu. Neutrophils release microvesicles containing AnxA1. These microvesicles dampen further neutrophil recruitment and induce macrophage phenotype switching toward a repair phenotype. Neutrophils express receptors such as CCR5 that can function as cytokine scavengers to reduce the availability of proinflammatory cytokines for other neutrophils. Neutrophils release NETs, which can trap proinflammatory chemokines.

such as LTB4. Indeed, following a tiny laser injury, fMLP played little to no role in neutrophil recruitment (37), whereas in a largerscale necrotic injury, fMLP played a dominant role (31).

Although it is conceivable that neutrophils respond to DAMPs or PAMPs in a similar fashion, growing evidence suggests that these signals can be integrated differently (38). An elegant study using zebrafish larvae showed that $\mathrm{H}_{2} \mathrm{O}_{2}$ was required for neutrophil recruitment to sterile injury but not infection with Pseudomonas aeruginosa (39). Along the same lines, in zebrafish, IL-1 $\beta$ signaling and myeloid differentiation primary response protein 88 (MYD88) signaling mediated neutrophil migration to sterile injury, but were not required for the neutrophil response to $E$. coli (40). Recently, a study in zebrafish demonstrated that recruitment of neutrophils to sterile injury was CXC chemokine receptor 1-mediated (CXCR1-mediated), while recruitment to infection required CXCR2 (41). Intravital imaging in the liver revealed that in a model of sepsis, neutrophils were actively recruited by interaction of neutrophil CD44 and endothelial hyaluronic acid (42). Conversely, in sterile injury, neutrophil adhesion was mediated by interaction of macrophage 1 antigen (MAC-1) and endothelial intercellular adhesion molecule 1 (ICAM-1), while further recruitment was orchestrated by CXC chemokine gradients followed by FPR-dependent danger signals from necrotic cells (ref. 31 and Figure 1). The brain is physiologically devoid of neutrophils, and in sterile brain injury, neutrophil recruitment was dependent on very late antigen 4 (VLA-4) (43), emphasizing neutrophils' utilization of different molecules to infiltrate different organs.

\section{Neutrophil-mediated tissue injury}

Considering the lethal cargo they carry to the site of perturbation, such as ROS and proteolytic and antimicrobial proteins, neutrophils have considerable potential to cause tissue damage $(44,45)$. Neutrophil contributions to tissue damage include the release of neutrophil-derived serine and matrix metalloproteases (MMPs) that cleave extracellular matrix (ECM) components, thereby destroying existing tissue architecture (46). In acute respiratory distress syndrome (ARDS), endothelial damage and vascular leakage was mediated by local release of elastase, MMPs, and ROS (47). In ischemic stroke, neutrophil accumulation and adherence to endothelium resulted in impaired perfusion (48), and ROS production was shown to directly damage endothelium and brain parenchyma (49). Extensive literature exists on the role of neutrophils and NETs in tissue damage (46, 50-53). Furthermore, neutrophils are also implicated in the pathogenesis of various chronic inflammatory conditions (17), such as rheumatoid arthritis (54), systemic lupus erythematosus (55), cancer (56), inflammatory bowel disease (57), atherosclerosis (58), and metabolic syndrome (59).

Neutrophils constantly face the predicament of eradicating pathogens while preserving tissue integrity, and this crucial balance might be influenced by circadian rhythm (60-62). Neutrophils liberated from bone marrow into the circulation undergo diurnal phenotypic changes termed "aging" (60). These aged neutrophils $\left(\mathrm{CD} 62 \mathrm{~L}^{\mathrm{lo}} \mathrm{CXCR} 4^{\text {hi }}\right)$ are repositioned from the circulation to tissues toward the end of their life cycle (60). Adrover et al. recently identified an intrinsic program mediating neutrophil aging, 
whereby Bmal1 (brain and muscle Arnt-like 1) regulated CXCR2 activation, resulting in diurnal transcriptional changes, and these changes were antagonized by CXCR4 (61). Using mouse models of constitutive neutrophil aging, the authors found that improving recruitment of aged neutrophils to tissues under homeostasis and having more neutrophils in tissues resulted in enhanced antimicrobial clearance in kidney infection. Furthermore, clearance of aged neutrophils from the vasculature protected from cardiac damage following myocardial infarction (MI), emphasizing the importance of diurnal neutrophil compartmentalization (61).

\section{Neutrophils in tissue repair}

In recent years it has become apparent that resolution of inflammation is an active process in which neutrophils play a seminal role. In most injuries, which are usually traumatic in nature, there is resolution involving controlled neutrophil activation and deactivation, while inflammation that involves an "unnatural stimulus," e.g., high-fat diets, alcohol, and drugs like acetaminophen or bleomycin, will lead to inadvertent and perpetual neutrophil activation and injury. In addition, ischemia/reperfusion associated with transplantation, or even MI and strokes, may also cause neutrophils to injure self. However, we would argue that as we have not evolved molecular mechanisms to deal with these perturbations, neutrophils have difficulty discerning and responding appropriately to these insults, resulting in their induction of maladaptive effector functions. Conversely, in trauma and other injuries, the neutrophil evolved a proper response to ensure survival, and absence of neutrophils might delay proper healing.

Neutrophils contribute to tissue repair via multiple mechanisms, and the response is often time-dependent (Figure 2 and ref. 45). As professional phagocytes, neutrophils are involved in clearing necrotic tissue and cellular debris. It is essential for tissue repair that cellular remnants are removed to prevent persistent proinflammatory signaling. It is unclear whether neutrophils also remove injured cells, but this would explain the many studies that show decreased area of injury if neutrophils are depleted before experimentation. The majority of neutrophil-depleting studies address acute injury without measuring repair that occurs days or weeks later, leaving the impression that neutrophils simply induce more injury. However, neutrophils contribute to resolution of inflammation and repair by releasing a plethora of presynthesized mediators such as growth factors and proangiogenic factors (63). A case in point is MMP-9, which is produced by neutrophils in great abundance and is capable of degrading DAMPs such as HMGB1 and HSP90, thus dampening the recruitment of additional inflammatory cells (64).

Angiogenesis is essential for healing, and new vasculature delivers oxygen and nutrients that facilitate tissue regrowth (65). Growing evidence suggests that neutrophils promote angiogenesis following injury to help in repair. Indeed, a subset of proangiogenic $\mathrm{CXCR}^{\text {hi}}{ }^{2}$ EGFR ${ }^{+} \mathrm{CD} 49 \mathrm{~d}^{+}$neutrophils was recently described in humans and mice $(66,67)$. In a model of avascular pancreatic islet transplantation, the neutrophils recruited by VEGF-A expression in pancreatic islet cells expressed high levels of MMP-9 (67), a potent activator of VEGF activity. MMP-9-deficient mice showed impaired revascularization (67). In a model of toxic corneal injury, neutrophils invading the cornea expressed high levels of VEGF
(68). Neutrophil depletion inhibited corneal angiogenesis and reduced protein levels of VEGF and the proinflammatory cytokines MIP- $1 \alpha$ and MIP-2. It has been proposed that neutrophils make the tunnels or sleeves that allow for revascularization into tissue in injured organs (69). Notably, the proangiogenic role of neutrophils extends further to other physiologic and pathophysiologic settings. A recent study identified a role for neutrophils in maintaining normal pregnancy and placental development by inducing proangiogenic T cells (70). Proangiogenic neutrophils were shown to promote tumor growth, perhaps through angiogenesis (71).

Intriguingly, all the toxic substances made by neutrophils, including NETs, oxidants, and proteases, may serve important healing functions. For example, neutrophil-mediated resolution was recently described in gout, a neutrophil-predominant joint inflammation. Surprisingly, this study showed that aggregation of NETs promoted resolution of inflammation by degrading cytokines and chemokines, thus disrupting the recruitment of additional inflammatory cells (ref. 72 and Figure 2). NETosis-deficient mice showed increased chronic inflammation, and adoptive transfer of in vitro aggregated NETs reduced this phenotype by degrading proinflammatory cytokines through NET-bound proteases (72). The idea of antiinflammatory NET functions warrants further validation, but is an intriguing concept. Much like NETs, oxidants can also have dual functions, and while their inflammatory properties are well documented, ROS can also suppress inflammation independent of their role in NETosis (52). In fact, even proteases have been shown to be both potently inflammatory and to play key roles in revascularization and healing (69).

Some investigators have attempted to explain the dichotomy of beneficial and detrimental roles of neutrophils with different neutrophil subsets, but the existence of neutrophil subsets and whether they reflect differential adaptation to local environments are unclear. Indeed, it was recently postulated that neutrophils can obtain different polarization states in the tumor microenvironment, leading to the terms "N1" (antitumorigenic) and "N2" (protumorigenic) (73), analogous to the M1/M2 concept in macrophages. Transition between N1 and N2 neutrophils was TGF- $\beta$ mediated, and transcriptomic analysis confirmed N1 and N2 neutrophils to be separate populations (74). Temporal neutrophil polarization was also recently shown following MI, with N2 neutrophils increasing during the healing phase (75). These studies discuss the possible existence of specialized repair neutrophils; however, lineage-tracing studies are needed to clarify the existence of bona fide neutrophil subsets (76).

It is quite intriguing that neutrophils in all organs have evolved to provide healing properties despite vast differences in architecture and specific tissue cell types. Nevertheless, below, we summarize the role of neutrophils as repair cells in various organs (Figure 3).

Neutrophils in lung repair. Early evidence indicated that neutrophil $\alpha$-defensins induce lung epithelial cell proliferation in vitro (77), and a recent study by Blázquez-Prieto et al. identified a role for neutrophils in lung repair via MMP-9 activity (78). The authors noted increased lung damage but reduced levels of MMP-9 in neutrophil-depleted mice with ventilator-associated lung injury. Similar findings were made in the bronchoalveolar lavage (BAL) fluid of neutropenic patients. Therapeutic adminis- 


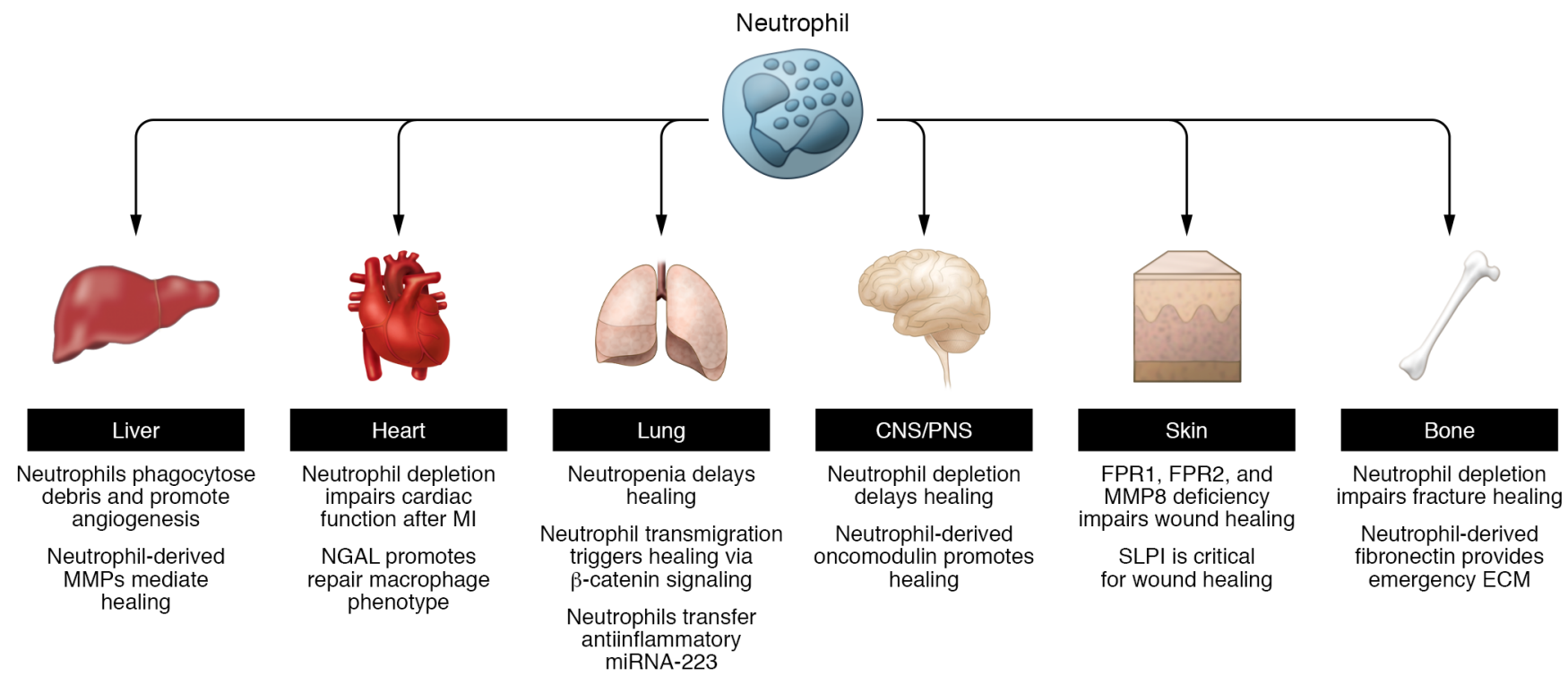

Figure 3. Schematic overview of studies highlighting neutrophil contributions to the repair of tissue injury in different organ systems. Neutrophils were shown to contribute to repair of tissue injury in different organ systems. Some mechanisms such as those promoting angiogenesis seem to be universal, whereas others seem to be restricted to certain organs. Examples shown include liver, heart, lung, CNS/PNS, skin, and bone fractures.

tration of MMP-9 significantly reduced tissue damage in acute lung injury (78). NADPH oxidase, a membrane-bound enzyme complex associated with intracellular membranes of phagosomes, known for its antimicrobial function through the production of ROS (79, 80 ), was recently discovered to also contribute to the attenuation of lung inflammation (81). NADPH oxidase-deficient mice developed progressive inflammation, augmented NF- $\mathrm{kB}$ activation, and elevated proinflammatory cytokines upon intratracheal zymosan or LPS challenge (81). In a subsequent study, the authors showed that NADPH oxidase deficiency led to increased neutrophil recruitment to the lungs following injury and that the NADPH oxidase's protective effect required activation of nuclear factor erythroid 2-related factor 2 (Nrf2), a redox-sensitive antiinflammatory transcription factor (82). Zemans et al. recently demonstrated that neutrophil transmigration across lung epithelial cells, in vivo in mice and in vitro using human neutrophils, triggered repair of lung epithelium via $\beta$-catenin signaling (83). Mice treated with intratracheal LPS showed activation of $\beta$-catenin signaling in type II pneumocytes, mediated by elastase-induced cleavage of E-cadherin, thus promoting epithelial repair (83). Further elaborating on these findings, Paris et al. demonstrated decreased re-epithelialization and increased alveolar protein concentration in neutrophildepleted or G-CSF-deficient mice in a model of acute lung injury mimicking ARDS (84). Unbiased proteomic analysis of BAL fluid revealed differential expression of MMP-2, MMP-9, and Fgf1, indicating that neutrophils could positively alter pathways related to epithelial regeneration (84). Neudecker et al. recently demonstrated another mechanism by which neutrophils promote tissue repair, elegantly showing that intercellular transfer of microRNAs (specifically miRNA-223) from neutrophils to pulmonary epithelial cells attenuated lung damage by repressing PARP-1 (85).

Neutrophils in central and peripheral nervous system repair. Regeneration after injury to the peripheral or central nervous sys- tem requires the immune system (86). In a model of spinal cord injury (SCI), Stirling et al. demonstrated a beneficial role for neutrophils (87) wherein neutrophil depletion delayed recovery, impaired wound healing, and reduced astrocyte reactivity (87). Moreover, secretory leukocyte protease inhibitor (SLPI), which is secreted by neutrophils and astrocytes in the spinal cord, was shown to be beneficial in SCI (88). Similarly, in a model of optic nerve injury, neutrophils produced high levels of the atypical growth factor oncomodulin, thereby contributing to optic nerve repair (89). Neutrophil depletion or an oncomodulin antagonist prevented optic nerve regeneration (89). It was recently shown that neutrophils play a crucial part in removal of debris following peripheral nerve injury (90). Neutrophil depletion significantly impaired clearance of nerve debris, demonstrating an important role for neutrophil-mediated phagocytosis as an initiating step of tissue repair (90).

Neutrophils in cutaneous wound healing. Neutrophils also play a crucial role in cutaneous wound repair, and neutropenic patients often suffer from impaired wound healing (44). Neutrophils are the first immune cells to arrive at cutaneous wounds in an effort to sterilize the injury (44). Liu et al. recently demonstrated a critical role for neutrophils in wound repair, as FPR1/2-deficient mice showed reduced neutrophil recruitment to wounds, which exhibited delayed healing (91). In a previous study, neutrophil depletion delayed wound healing in older mice but not young mice, an effect that was reversed in older mice upon G-CSF injections (92). In another study, MMP-8-deficient mice displayed delayed healing and reduced neutrophil infiltration at early stages but persistent inflammation at later stages (93). Mechanistically, TGF- $\beta$ signaling and neutrophil apoptosis were impaired in MMP-8-deficient mice (93). Moreover, human neutrophils that migrated to skin wounds showed transcriptional differences and upregulated levels of cytokines and chemokines that promote angiogenesis and keratinocyte and fibroblast proliferation compared with circulat- 
ing neutrophils (94). This could perhaps be related to the transmigration into a new environment. Cutaneous neutrophils also produce SLPI, and SLPI-deficient mice showed increased levels of inflammation and elastase as well as delayed wound healing (95). Chronic wounds have also been associated with increased protease levels, reduced neutrophil apoptosis, and neutrophil persistence, indicating that signals from the wound environment likely contribute to the fate of neutrophils (44). Interestingly, in diabetes, neutrophils were primed to undergo NETosis impairing normal wound healing in a high-glucose environment (96).

Neutrophils in cardiovascular repair. $\mathrm{MI}$ is accompanied by a pronounced neutrophilic infiltration, and the prevailing view has been that neutrophils augment cardiac damage (97). However, an elegant study recently demonstrated that neutrophil depletion led to impaired cardiac function, increased fibrosis, and progressive heart failure following MI (98). Mechanistically, the authors found altered macrophage polarization states in neutrophil-depleted mice. Furthermore, neutrophil gelatinase-associated lipocalin (NGAL) seemed to increase the capacity of cardiac macrophages to engulf apoptotic cells, and treatment with recombinant NGAL was able to restore this phenotype in neutrophil-depleted mice (98). A previous study identified that the cytokine oncostatin M (OSM), produced by neutrophils and macrophages, induced dedifferentiating cardiomyocytes to release regenerating islet-derived protein $3 \beta$ (REG3 $\beta$ ), which then modulated the degree of macrophage accumulation in the heart to fine-tune wound healing (99). Furthermore, neutrophilborne cathelicidin (mouse CRAMP, human LL-37) was recently discovered to mediate arterial healing by promoting re-endothelialization after acute injury in mice and patients (100).

Neutrophils in bone fracture healing. The role of neutrophils in bone fracture healing is well recognized (101-103). Fracture healing is characterized by an inflammatory phase, a repair phase, and ultimately a remodeling phase (104). The initial inflammatory response starts with a fracture hematoma that serves as a scaffold for immune cells and progenitor cells (104). Neutrophils are found in abundance in the fracture hematoma, arriving within minutes after fracture, and comprise both mature neutrophils from the circulation and immature neutrophils from bone marrow (102). G-CSF applied locally to fractures was associated with a significant improvement in healing and angiogenesis (105). In a mouse model of bone fractures, the authors noted that depletion of neutrophils led to impaired healing after fracture (106). Furthermore, they observed increased levels of inflammatory cytokines as well as altered monocyte and macrophage recruitment, indicating a crucial role for neutrophils in initiating the repair process. Along those lines, Bastian et al. recently discovered neutrophils in the fracture hematoma of human patients (107). These neutrophils costained with fibronectin, and hence this group proposed a mechanism of "emergency ECM production" by infiltrating neutrophils promoting early fracture healing (107).

Neutrophils in liver repair. The liver is an organ with a remarkable regenerative capacity and thus is often used to scrutinize tissue repair experimentally. Numerous studies suggest neutrophil involvement in the pathogenesis of acute and chronic liver diseases (108-110). A fully healing model of thermal liver injury demonstrated that neutrophils, guided by an intravascular chemokine gradient, were the first cells to arrive at the injury site, where they infiltrated the necrotic area (ref. 31 and Figure 1). Interestingly, neutrophil depletion resulted in increased cellular debris, delayed revascularization, and ultimately delayed healing (69). Intravital imaging revealed that neutrophils contributed to healing by phagocytosing debris and promoting vascular regrowth in part by making new sleeves for blood vessels to grow into (69). Along the same lines, a recent study identified a dual role for neutrophils in acetaminophen-induced acute liver injury, with neutrophil-mediated injury amplification early on, but protective effects during the repair phase, as depletion increased liver damage (111). The early phase may reflect killing and/or removal of injured cells that presents as increased injury but is required for repair. A recent study by Saijou et al. (112) investigated the role of neutrophils in chronic liver injury. Using a mouse deficient in tribbles pseudokinase (Trib1), which increases neutrophil counts, the authors found reduced liver fibrosis, increased intrahepatic neutrophils, and increased MMP-8 and MMP-9 levels. Neutrophil infusion diminished fibrosis, while neutrophil depletion increased fibrosis (112). As a result, the authors concluded that neutrophils suppressed fibrosis in chronic liver injury potentially by promoting fibrolysis through the production of metalloproteases (112). Along the same lines, in chronic cholestatic liver injury, neutrophil depletion decreased MMP-8 levels and decreased collagen degradation (113). Clearly, in liver, neutrophils are very important in regulating regeneration versus fibrosis.

All in all, it is evident that regardless of the tissue, neutrophils contribute to healing. Some of the mechanisms, such as MMP delivery, appear to be nonspecific and occur in all organs, while in certain situations, including healing of the optic nerve, or regrowth of lung epithelial cells, there are clear specific effector functions.

\section{Resolution of neutrophilic inflammation}

Once their job is done, neutrophils must be removed from the injury site. Indeed, neutrophil clearance from the inflicted tissue is essential to induce a pro-resolution cascade (45). Clearance can occur either by apoptosis/necrosis and subsequent engulfment by macrophages (efferocytosis) or by overt egress of neutrophils from the tissue site, either via reverse migration back into the vasculature or via expulsion to the external environment (as may occur in the intestinal tract, skin, and other mucosal tissues). Apoptotic neutrophils expose phosphatidylserine on their surface, and their clearance by macrophages induces a pro-resolving cascade, including the release of repair cytokines such as TGF- $\beta$, IL-10, and VEGF, leading to macrophages reprogramming to an antiinflammatory phenotype (refs. 45, 114, and Figure 1). In addition, macrophages release resolvins and lipoxins, further enhancing efferocytosis (63). Tissue-resident macrophages are a major source of TNF- $\alpha$ in the inflammatory milieu. At lower concentrations, TNF- $\alpha$ promotes neutrophil survival; however, at higher concentrations, TNF- $\alpha$ has proapoptotic effects on neutrophils (115). Furthermore, the release of $\alpha$-defensins by apoptotic neutrophils increases the phagocytic capacity of macrophages and dampens their release of inflammatory mediators (116).

Cells in the inflamed tissue produce pro-resolving lipid mediators such as lipoxin A4 (LXA4) $(4,117,118)$. LXA4 inhibits neutrophil migration and promotes efferocytosis by macrophages (119). Moreover, neutrophils and other cells in the inflammatory milieu produce specialized pro-resolving mediators, including resolvins 
and protectins, which stop neutrophil migration at later stages by interfering with chemotactic signals (120). Upregulation of CC chemokine receptor 5 (CCR5) was shown to scavenge proinflammatory cytokines, reducing their availability for additional neutrophils (121). In summary, pro-resolving lipid mediators decrease vascular permeability, inhibit further neutrophil recruitment, induce neutrophil apoptosis, and promote efferocytosis at sites of inflammation, thus triggering a resolving cascade (63).

Microvesicles (also known as microparticles or ectosomes) are extracellular membrane vesicles initially identified as a mechanism of cell-cell communication $(122,123)$ and have since been implicated as a neutrophil-mediated killing mechanism to restrict bacterial growth (124). Moreover, microvesicles were shown to be involved in the pro-resolving role of neutrophils. Release of microvesicles exposes phosphatidylserine on the cell surface, which promotes antiinflammatory cytokine release by macrophages and dendritic cells (125-127). A recent study using isolated human neutrophils cocultured with macrophages in vitro demonstrated that TNF- $\alpha$ stimulation generated microvesicles from neutrophils, exposing phosphatidylserine and annexin A1, which in turn induced macrophage release of TGF- $\beta$ (125). Another study described a protective role for neutrophil microvesicles in arthritis (128). Administration of annexin A1-containing microvesicles promoted arthritis recovery, and mice with impaired microvesicle release showed more severe arthritis. In mice, IL-10 production by neutrophils seems to contribute to resolution of injury, but current evidence argues against human neutrophils as a source of IL-10, suggesting that this mechanism is not common to all mammals (129-132).

As mentioned, annexin A1 is a pro-resolving protein stored in large amounts in neutrophil granules (63). Glucocorticoids, potent antiinflammatory drugs, operate by increasing annexin A1 synthesis (133) and promote the relocation of annexin A1 to the cell surface, leading to neutrophil apoptosis, which increases the capacity of macrophage efferocytosis (134-136). Recently, a study described monocyte recruitment driven by neutrophil-derived annexin A1 in a model of peritonitis, leading to antiinflammatory cytokine release and resolution of inflammation (137).

Clearly there is much evidence to support the view that neutrophils undergo apoptosis at the site of injury and are subsequently cleared by local macrophages. Indeed, persistence of neutrophils results in additional tissue damage and chronic inflammation. However, a number of issues remain unresolved. In various models of sterile inflammation, neutrophils entered and disappeared well before monocytes were recruited, and depletion of monocytes and/or macrophages had no impact on neutrophil disappearance (69). In addition, intravital microscopy carefully tracking both neutrophils and mononuclear cells failed to show any overt phagocytosis of neutrophils by mononuclear cells (69). Indeed, the study revealed that neutrophils migrated back into the vasculature, in a process known as reverse transmigration (24, 69). The first in vivo evidence of reverse transmigration was provided by Mathias et al., who demonstrated neutrophils migrating away from a wound back to the vasculature using intravital imaging in zebrafish (138). Similarly, human neutrophils were found to display bidirectional movements through endothelial monolayers in vitro (139). Neutrophils undergoing reverse transendothelial migration had a distinct phenotype (ICAM- $1^{\mathrm{hi}} \mathrm{CXCR} 1^{\mathrm{lo}}$ ), and in patients with systemic inflammation this population was increased. In subsequent studies in mice, neutrophils were also shown to exhibit reverse transmigration in ischemia/reperfusion injury. This process was dependent on the ability of neutrophils to proteolytically degrade the junctional adhesion molecule JAM-C (140). Additional work concluded that neutrophil elastase was critically involved to promote transendothelial migration in mice by degrading JAM-C (141). Reverse migration was also reported in a model of focal hepatic sterile injury, but in this case the neutrophils initially performed important repair functions before returning back to the vasculature (ref. 69 and Figure 1). Neutrophils at the injury border showed directional movement away from the injury; however, the chemotactic cues involved were not elucidated. Using photoactivation, neutrophils were observed migrating from the liver parenchyma back into the vasculature, stopping in the capillaries of the lung, where they upregulated CXCR4, and ultimately homing back to the bone marrow, presumably dying via apoptosis (ref. 69 and Figure 1). Interestingly, mice deficient in cathepsin C (CTSC) (rendering them unable to activate various proteases) had normal numbers of neutrophils migrating to the injury but fewer neutrophils leaving the injury, and this disrupted the normal revascularization process (69).

Reverse transmigration may explain why neutrophils end up causing ARDS following severe injuries. One could imagine that a massive injury, wherein many neutrophils reverse-transmigrated, could lead to systemic inflammation. Indeed, in mice and humans with acute pancreatitis, neutrophils with a reverse transmigration phenotype were found in both the peripheral blood and the lungs and correlated with severity of lung injury (142). JAM-C-deficient mice had higher numbers of reverse-transmigrated neutrophils in blood and lung vasculature and increased lung injury. The authors hypothesized that pancreatic downregulation of JAM-C permitted neutrophil reverse transmigration, overwhelming the lung.

Molecular mechanisms of reverse transmigration are largely unresolved. In zebrafish, the HIF-1 $\alpha$ pathway was implicated in neutrophil retention at wound sites (143), and as this molecule disappears there may be fewer retained neutrophils within the injury site. High CXCL8 concentrations have been shown to function as a chemorepellent (144). An intriguing study recently demonstrated that CXCL8a/CXCR2 signaling promotes reverse migration of neutrophils in sterile injury in vivo in zebrafish and in human neutrophils (41). Collectively, these studies suggest that reverse migration contributes to the resolution of inflammation, albeit with the possible caveat that during severe injury this could cause additional inflammation elsewhere. Many questions remain regarding the precise mechanisms and cues that dictate reverse migration versus apoptosis as well as the presence of this phenomenon in humans in vivo. Considering the billions of neutrophils produced daily, it is remarkable that their exact turnover in inflammatory settings as well as in homeostasis is still poorly understood. To that end, the proportion of neutrophils that are phagocytosed, reverse-migrated, or cleared to the external environment is enigmatic and warrants more investigation (20).

\section{Concluding remarks}

Timely and effective tissue repair after injury is of utmost importance and as such has been optimized to perfection over millions 
of years of evolution. The immune system is employed as an integral machinery to drive tissue repair. Inflammation following sterile injury, such as trauma, is characterized by an initial hyperinflammatory phase aimed at eliminating potential microbial invaders and clearing the injury site of debris. In this early phase, neutrophil-induced tissue damage might be inevitable in light of the important task at hand and might even be necessary to initiate proper tissue restoration. Neutrophils phagocytose cellular debris but then importantly contribute to neovascularization and promote normal collagen deposition and remodeling $(69,145)$. The view of neutrophils as unrestrained killers seems evolutionarily flawed, and we would argue that neutrophils both partake in the proinflammatory response to tissue injury and also pave the way for its antiinflammatory and repair phases. Whether these phases are mediated by different polarization states of the neutrophil or bona fide pro- and antiinflammatory subsets remains unclear. Ultimately, it is essential for neutrophils to leave the site either by apoptosis or by reverse transmigration. Failure to clear the space of neutrophils may lead to disrepair and chronic inflammation. In a perturbed system, such as diabetic ulcer wounds, neutrophils might actually enhance damage as a result of an undermined optimal tissue repair cascade and alterations in necessary cues.

We believe targeting neutrophils at different phases of inflammation might eventually show promising results - but our understanding of the fine temporal and spatial dynamics of these remarkable cells is still incomplete and warrants further investi- gation. Uncovering the precise cues that distinguish self-limiting inflammation from chronic inflammation and identifying the neutrophil's contribution will be crucial to improve current therapies. Finally, much of the current literature depends on animal models, since it is difficult to do these experiments in humans. Nevertheless, impaired wound healing has been described as a clinical feature of congenital neutrophil disorders in humans $(146,147)$. Case reports also showed wound healing improvements in neutropenic patients receiving G-CSF $(148,149)$, and in one study assessing dental extraction in neutropenic patients, delayed wound healing was the most prevalent complication (150). As such, the limited human data do support a role for neutrophils as players in wound healing and warrant attention in the development of strategies to improve wound repair.

\section{Acknowledgments}

MP is supported by the German Research Foundation (DFG) with a postdoctoral research fellowship (PE 2737/1-1). PK is supported by grants from the Canadian Institute of Health Research, the Heart and Stroke Foundation of Canada, and the Canada Research Chairs Program. The authors thank Bas Surewaard for carefully proofreading the manuscript.

Address correspondence to: Paul Kubes, University of Calgary, 3330 Hospital Drive NW, Calgary, Alberta T2N 4N1, Canada. Phone: 403.220.2705; Email: pkubes@ucalgary.ca.
1. Amulic B, Cazalet C, Hayes GL, Metzler KD, Zychlinsky A. Neutrophil function: from mechanisms to disease. Annu Rev Immunol. 2012;30:459-489.

2. Phillipson M, Kubes P. The neutrophil in vascular inflammation. Nat Med. 2011;17(11):1381-1390.

3. Meyer E, et al. Risk factor analysis of blood stream infection and pneumonia in neutropenic patients after peripheral blood stem-cell transplantation. Bone Marrow Transplant. 2007;39(3):173-178.

4. Kolaczkowska E, Kubes P. Neutrophil recruitment and function in health and inflammation. Nat Rev Immunol. 2013;13(3):159-175.

5. Ehrlich P. Methodologische Beiträge zur Physiologie und Pathologie der verschiedenen Formen der Leukocyten. Z Klin Med. 1880;1:553-560.

6. Metchnikoff E. Lecon sur la pathologie comparee de inflammation. Ann Inst Pasteur. 1893;7:348-357.

7. Kaufmann SHE. Immunology's foundation: the 100-year anniversary of the Nobel Prize to Paul Ehrlich and Elie Metchnikoff. Nat Immunol. 2008;9(7):705-712.

8. Campbell EJ, Senior RM, McDonald JA, Cox DL. Proteolysis by neutrophils. Relative importance of cell-substrate contact and oxidative inactivation of proteinase inhibitors in vitro. JClin Invest. 1982;70(4):845-852.

9. Nicolás-Ávila JÁ, Adrover JM, Hidalgo A. Neutrophils in homeostasis, immunity, and cancer. Immunity. 2017;46(1):15-28.

10. Brinkmann V, et al. Neutrophil extracellular traps kill bacteria. Science. 2004;303(5663):1532-1535

11. Yipp BG, Kubes P. NETosis: how vital is it? Blood. 2013;122(16):2784-2794.
12. Ley K, et al. Neutrophils: new insights and open questions. Sci Immunol. 2018;3(30):eaat4579.

13. Hasenberg A, et al. Catchup: a mouse model for imaging-based tracking and modulation of neutrophil granulocytes. Nat Methods. 2015;12(5):445-452.

14. Casanova-Acebes M, et al. Neutrophils instruct homeostatic and pathological states in naive tissues. J Exp Med. 2018;215(11):2778-2795.

15. Puga I, et al. B cell-helper neutrophils stimulate the diversification and production of immunoglobulin in the marginal zone of the spleen. Nat Immunol. 2011;13(2):170-180.

16. Hampton HR, Bailey J, Tomura M, Brink R, Chtanova T. Microbe-dependent lymphatic migration of neutrophils modulates lymphocyte proliferation in lymph nodes. Nat Commun. 2015;6:7139.

17. Soehnlein O, Steffens S, Hidalgo A, Weber C. Neutrophils as protagonists and targets in chronic inflammation. Nat Rev Immunol. 2017;17(4):248-261

18. Zhang D, et al. Neutrophil ageing is regulated by the microbiome. Nature. 2015;525(7570):528-532

19. Coffelt SB, Wellenstein MD, de Visser KE. Neutrophils in cancer: neutral no more. Nat Rev Cancer. 2016;16(7):431-446.

20. Kubes P. The enigmatic neutrophil: what we do not know. Cell Tissue Res. 2018;371(3):399-406.

21. Ley K. Integration of inflammatory signals by rolling neutrophils. Immunol Rev. 2002;186:8-18.

22. Futosi K, Fodor S, Mócsai A. Reprint of neutrophil cell surface receptors and their intracellular signal transduction pathways. Int Immunopharmacol. 2013;17(4):1185-1197.

23. Pittman K, Kubes P. Damage-associated molecu- lar patterns control neutrophil recruitment. J Innate Immun. 2013;5(4):315-323.

24. de Oliveira S, Rosowski EE, Huttenlocher A. Neutrophil migration in infection and wound repair: going forward in reverse. Nat Rev Immunol. 2016;16(6):378-391.

25. Sadik CD, Kim ND, Luster AD. Neutrophils cascading their way to inflammation. Trends Immunol. 2011;32(10):452-460.

26. Ley K, Laudanna C, Cybulsky MI, Nourshargh S. Getting to the site of inflammation: the leukocyte adhesion cascade updated. Nat Rev Immunol. 2007;7(9):678-689.

27. de Oliveira S, López-Muñoz A, Candel S, Pelegrín P, Calado Â, Mulero V. ATP modulates acute inflammation in vivo through dual oxidase 1-derived $\mathrm{H} 2 \mathrm{O} 2$ production and $\mathrm{NF}-\kappa \mathrm{B}$ activation. J Immunol. 2014;192(12):5710-5719.

28. Chen Y, et al. ATP release guides neutrophil chemotaxis via $\mathrm{P} 2 \mathrm{Y} 2$ and $\mathrm{A} 3$ receptors. Science. 2006;314(5806):1792-1795.

29. Zhang Q, et al. Circulating mitochondrial DAMPs cause inflammatory responses to injury. Nature. 2010;464(7285):104-107.

30. Li L, et al. New development in studies of formyl-peptide receptors: critical roles in host defense. J Leukoc Biol. 2016;99(3):425-435.

31. McDonald B, et al. Intravascular danger signals guide neutrophils to sites of sterile inflammation. Science. 2010;330(6002):362-366.

32. Dorward DA, et al. Novel role for endogenous mitochondrial formylated peptide-driven formyl peptide receptor 1 signalling in acute respiratory distress syndrome. Thorax. 2017;72(10):928-936.

33. Petri MH, Laguna-Fernández A, Gonzalez-Diez 
M, Paulsson-Berne G, Hansson GK, Bäck M. The role of the FPR2/ALX receptor in atherosclerosis development and plaque stability. Cardiovasc Res. 2015;105(1):65-74.

34. Drechsler M, et al. Annexin A1 counteracts chemokine-induced arterial myeloid cell recruitment. Circ Res. 2015;116(5):827-835.

35. Döring Y, et al. Lack of neutrophil-derived CRAMP reduces atherosclerosis in mice. Circ Res. 2012;110(8):1052-1056.

36. Lämmermann T. In the eye of the neutrophil swarm-navigation signals that bring neutrophils together in inflamed and infected tissues. J Leukoc Biol. 2016;100(1):55-63.

37. Lämmermann $\mathrm{T}$, et al. Neutrophil swarms require LTB 4 and integrins at sites of cell death in vivo. Nature. 2013;498(7454):371-375.

38. Tan RS, Ho B, Leung BP, Ding JL. TLR cross-talk confers specificity to innate immunity. Int Rev Immunol. 2014;33(6):443-453.

39. Deng Q, Harvie EA, Huttenlocher A. Distinct signalling mechanisms mediate neutrophil attraction to bacterial infection and tissue injury. Cell Microbiol. 2012;14(4):517-528.

40. Yan B, et al. IL-1 $\beta$ and reactive oxygen species differentially regulate neutrophil directional migration and basal random motility in a zebrafish injury-induced inflammation model. J Immunol. 2014;192(12):5998-6008.

41. Powell D, Tauzin S, Hind LE, Deng Q, Beebe DJ, Huttenlocher A. Chemokine signaling and the regulation of bidirectional leukocyte migration in interstitial tissues. Cell Rep. 2017;19(8):1572-1585.

42. McDonald B, et al. Interaction of CD44 and hyaluronan is the dominant mechanism for neutrophil sequestration in inflamed liver sinusoids. JExp Med. 2008;205(4):915-927.

43. Neumann J, et al. Very-late-antigen-4 (VLA-4)-mediated brain invasion by neutrophils leads to interactions with microglia, increased ischemic injury and impaired behavior in experimental stroke. Acta Neuropathol. 2015;129(2):259-277.

44. Wilgus TA, Roy S, McDaniel JC. Neutrophils and wound repair: positive actions and negative reactions. Adv Wound Care (New Rochelle). 2013;2(7):379-388.

45. Wang J. Neutrophils in tissue injury and repair. Cell Tissue Res. 2018;371(3):531-539.

46. Kruger P, et al. Neutrophils: between host defence, immune modulation, and tissue injury. PLoS Pathog. 2015;11(3):e1004651.

47. Ware L. Pathophysiology of acute lung injury and the acute respiratory distress syndrome. Semin Respir Crit Care Med. 2006;27(4):337-349.

48. Mori E, del Zoppo GJ, Chambers JD, Copeland $\mathrm{BR}$, Arfors KE. Inhibition of polymorphonuclear leukocyte adherence suppresses no-reflow after focal cerebral ischemia in baboons. Stroke. 1992;23(5):712-718.

49. Chen H, Song YS, Chan PH. Inhibition of NADPH oxidase is neuroprotective after ischemia - reperfusion. J Cereb Blood Flow Metab. 2009;29(7):1262-1272.

50. Segel GB, Halterman MW, Lichtman MA. The paradox of the neutrophil's role in tissue injury. JLeukoc Biol. 2011;89(3):359-372.

51. Fujishima S, Aikawa N. Neutrophil-mediated tis- sue injury and its modulation. Intensive Care Med. 1995;21(3):277-285.

52. Papayannopoulos V. Neutrophil extracellular traps in immunity and disease. Nat Rev Immunol. 2018;18(2):134-147.

53. Otxoa-de-Amezaga A, et al. Microglial cell loss after ischemic stroke favors brain neutrophil accumulation. Acta Neuropathol. 2019;137(2):321-341.

54. Camps M, et al. Blockade of PI3Kgamma suppresses joint inflammation and damage in mouse models of rheumatoid arthritis. Nat Med. 2005;11(9):936-943.

55. Pisitkun $P$, et al. Interleukin-17 cytokines are critical in development of fatal lupus glomerulonephritis. Immunity. 2012;37(6):1104-1115.

56. Coffelt SB, Wellenstein MD, de Visser KE. Neutrophils in cancer: neutral no more. Nat Rev Cancer. 2016;16(7):431-446.

57. Honda M, Kubes P. Neutrophils and neutrophil extracellular traps in the liver and gastrointestinal system. Nat Rev Gastroenterol Hepatol. 2018;15(4):206-221.

58. Soehnlein O. Multiple roles for neutrophils in atherosclerosis. Circ Res. 2012;110(6):875-888.

59. Talukdar $\mathrm{S}$, et al. Neutrophils mediate insulin resistance in mice fed a high-fat diet through secreted elastase. Nat Med. 2012;18(9):1407-1412.

60. Casanova-Acebes M, et al. Rhythmic modulation of the hematopoietic niche through neutrophil clearance. Cell. 2013;153(5):1025-1035.

61. Adrover JM, et al. A neutrophil timer coordinates immune defense and vascular protection. Immunity. 2019;50(2):390-402.e10.

62. Reglero-Real N, Rolas L, Nourshargh S. Leukocyte trafficking: time to take time seriously. Immunity. 2019;50(2):273-275.

63. Jones HR, Robb CT, Perretti M, Rossi AG. The role of neutrophils in inflammation resolution. Semin Immunol. 2016;28(2):137-145.

64. Cauwe B, Martens E, Proost P, Opdenakker G. Multidimensional degradomics identifies systemic autoantigens and intracellular matrix proteins as novel gelatinase B/MMP-9 substrates. Integr Biol (Camb). 2009;1(5-6):404-426.

65. DiPietro LA. Angiogenesis and scar formation in healing wounds. Curr Opin Rheumatol. 2013;25(1):87-91.

66. Massena S, et al. Identification and characterization of VEGF-A-responsive neutrophils expressing CD49d, VEGFR1, and CXCR4 in mice and humans. Blood. 2015;126(17):2016-2026.

67. Christoffersson G, et al. VEGF-A recruits a proangiogenic MMP-9-delivering neutrophil subset that induces angiogenesis in transplanted hypoxic tissue. Blood. 2012;120(23):4653-4662.

68. Gong Y, Koh DR. Neutrophils promote inflammatory angiogenesis via release of preformed VEGF in an in vivo corneal model. Cell Tissue Res. 2010;339(2):437-448

69. Wang J, Hossain M, Thanabalasuriar A, Gunzer $\mathrm{M}$, Meininger C, Kubes P. Visualizing the function and fate of neutrophils in sterile injury and repair. Science. 2017;358(6359):111-116.

70. Nadkarni S, et al. Neutrophils induce proangiogenic $\mathrm{T}$ cells with a regulatory phenotype in pregnancy. Proc Natl Acad Sci U S A. 2016;113(52):E8415-E8424.
71. Jablonska J, Leschner S, Westphal K, Lienenklaus $\mathrm{S}$, Weiss S. Neutrophils responsive to endogenous IFN- $\beta$ regulate tumor angiogenesis and growth in a mouse tumor model. JClin Invest. 2010;120(4):1151-1164.

72. Schauer C, et al. Aggregated neutrophil extracellular traps limit inflammation by degrading cytokines and chemokines. Nat Med.2014;20(5):511-517.

73. Fridlender ZG, et al. Polarization of tumor-associated neutrophil phenotype by TGF- $\beta$ : "N1" versus "N2" TAN. Cancer Cell. 2009;16(3):183-194.

74. Shaul ME, et al. Tumor-associated neutrophils display a distinct N1 profile following TGF $\beta$ modulation: a transcriptomics analysis of pro- vs. antitumor TANs. Oncoimmunology. 2016;5(11):e1232221.

75. Ma Y, et al. Temporal neutrophil polarization following myocardial infarction. Cardiovasc Res. 2016;110(1):51-61.

76. Deniset JF, Kubes P. Neutrophil heterogeneity: bona fide subsets or polarization states? J Leukoc Biol. 2018;103(5):829-838.

77. Aarbiou J, et al. Human neutrophil defensins induce lung epithelial cell proliferation in vitro. J Leukoc Biol. 2002;72(1):167-174.

78. Blázquez-Prieto J, et al. Impaired lung repair during neutropenia can be reverted by matrix metalloproteinase-9. Thorax. 2018;73(4):321-330.

79. Nguyen GT, Green ER, Mecsas J. Neutrophils to the ROScue: mechanisms of NADPH oxidase activation and bacterial resistance. Front Cell Infect Microbiol. 2017;7:373.

80. Groemping Y, Rittinger K. Activation and assembly of the NADPH oxidase: a structural perspective. Biochem J. 2005;386(pt 3):401-416.

81. Segal BH, et al. NADPH oxidase limits innate immune responses in the lungs in mice. PLoS One. 2010;5(3):e9631.

82. Davidson BA, et al. NADPH oxidase and Nrf2 regulate gastric aspiration-induced inflammation and acute lung injury. J Immunol. 2013;190(4):1714-1724.

83. Zemans RL, et al. Neutrophil transmigration triggers repair of the lung epithelium via $\beta$-catenin signaling. Proc Natl Acad Sci U S A. 2011;108(38):15990-15995.

84. Paris AJ, et al. Neutrophils promote alveolar epithelial regeneration by enhancing type II pneumocyte proliferation in a model of acid-induced acute lung injury. Am J Physiol Lung Cell Mol Physiol. 2016;311(6):L1062-L1075.

85. Neudecker V, et al. Neutrophil transfer of $m i R-223$ to lung epithelial cells dampens acute lung injury in mice. Sci Transl Med. 2017;9(408):eaah5360.

86. Donnelly DJ, Popovich PG. Inflammation and its role in neuroprotection, axonal regeneration and functional recovery after spinal cord injury. Exp Neurol. 2008;209(2):378-388.

87. Stirling DP, Liu S, Kubes P, Yong VW. Depletion of Ly6G/Gr-1 leukocytes after spinal cord injury in mice alters wound healing and worsens neurological outcome. J Neurosci. 2009;29(3):753-764.

88. Ghasemlou N, et al. Beneficial effects of secretory leukocyte protease inhibitor after spinal cord injury. Brain. 2010;133(pt 1):126-138.

89. Kurimoto T, et al. Neutrophils express oncomodulin and promote optic nerve regeneration. JNeurosci. 2013;33(37):14816-14824. 
90. Lindborg JA, Mack M, Zigmond RE. Neutrophils are critical for myelin removal in a peripheral nerve injury model of wallerian degeneration. J Neurosci. 2017;37(43):10258-10277.

91. Liu M, et al. Formylpeptide receptors mediate rapid neutrophil mobilization to accelerate wound healing. PLoS One. 2014;9(6):e90613.

92. Nishio N, Okawa Y, Sakurai H, Isobe K. Neutrophil depletion delays wound repair in aged mice. Age (Dordr). 2008;30(1):11-19.

93. Gutiérrez-Fernández A, et al. Increased inflammation delays wound healing in mice deficient in collagenase-2 (MMP-8). FASEB J. 2007;21(10):2580-2591.

94. Theilgaard-Mönch K, Knudsen S, Follin P, Borregaard $\mathrm{N}$. The transcriptional activation program of human neutrophils in skin lesions supports their important role in wound healing. JImmunol. 2004;172(12):7684-7693.

95. Ashcroft GS, et al. Secretory leukocyte protease inhibitor mediates non-redundant functions necessary for normal wound healing. Nat Med. 2000;6(10):1147-1153.

96. Wong SL, et al. Diabetes primes neutrophils to undergo NETosis, which impairs wound healing. Nat Med.2015;21(7):815-819.

97. Ma Y, Yabluchanskiy A, Lindsey ML. Neutrophil roles in left ventricular remodeling following myocardial infarction. Fibrogenesis Tissue Repair. 2013;6(1):11.

98. Horckmans M, et al. Neutrophils orchestrate post-myocardial infarction healing by polarizing macrophages towards a reparative phenotype. Eur Heart J. 2017;38(3):187-197.

99. Lörchner $\mathrm{H}$, et al. Myocardial healing requires Reg $3 \beta$-dependent accumulation of macrophages in the ischemic heart. Nat Med. 2015;21(4):353-362.

100.Soehnlein O, et al. Neutrophil-derived cathelicidin protects from neointimal hyperplasia. $\mathrm{Sci}$ Transl Med. 2011;3(103):103ra98.

101.Loi F, Córdova LA, Pajarinen J, Lin TH, Yao Z, Goodman SB. Inflammation, fracture and bone repair. Bone. 2016;86:119-130.

102. Kovtun A, Messerer DAC, Scharffetter-Kochanek K, Huber-Lang M, Ignatius A. Neutrophils in tissue trauma of the skin, bone, and lung: two sides of the same coin. JImmunol Res. 2018;2018:8173983.

103. Einhorn TA, Gerstenfeld LC. Fracture healing: mechanisms and interventions. Nat Rev Rheumatol. 2015;11(1):45-54

104. Claes L, Recknagel S, Ignatius A. Fracture healing under healthy and inflammatory conditions. Nat Rev Rheumatol. 2012;8(3):133-143.

105. Ishida K, et al. Bone regeneration properties of granulocyte colony-stimulating factor via neovascularization and osteogenesis. Tissue Eng Part A. 2010;16(10):3271-3284.

106. Kovtun A, Bergdolt S, Wiegner R, Radermacher P, Huber-Lang M, Ignatius A. The crucial role of neutrophil granulocytes in bone fracture healing. Eur Cell Mater. 2016;32:152-162.

107. Bastian OW, Koenderman L, Alblas J, Leenen LP, Blokhuis TJ. Neutrophils contribute to fracture healing by synthesizing fibronectin+ extracellular matrix rapidly after injury. Clin Immunol. 2016;164:78-84
108. Jaeschke H, Farhood A, Smith CW. Neutrophils contribute to ischemia/reperfusion injury in rat liver in vivo. FASEB J. 1990;4(15):3355-3359.

109. Xu R, Huang H, Zhang Z, Wang FS. The role of neutrophils in the development of liver diseases. Cell Mol Immunol. 2014;11(3):224-231.

110.Liu Z-X, Han D, Gunawan B, Kaplowitz N. Neutrophil depletion protects against murine acetaminophen hepatotoxicity. Hepatology. 2006;43(6):1220-1230.

111. Alvarenga DM, et al. Paradoxical role of matrix metalloproteinases in liver injury and regeneration after sterile acute hepatic failure. Cells. 2018;7(12):247.

112. Saijou E, et al. Neutrophils alleviate fibrosis in the CCl4-induced mouse chronic liver injury model. Hepatol Commun. 2018;2(6):703-717.

113. Harty MW, et al. Neutrophil depletion blocks early collagen degradation in repairing cholestatic rat livers. Am J Pathol. 2010;176(3):1271-1281.

114. Soehnlein O, Lindbom L. Phagocyte partnership during the onset and resolution of inflammation. Nat Rev Immunol. 2010;10(6):427-439.

115. van den Berg JM, Weyer S, Weening JJ, Roos D, Kuijpers TW. Divergent effects of tumor necrosis factor $\alpha$ on apoptosis of human neutrophils. J Leukoc Biol. 2001;69(3):467-473.

116. Miles K, et al. Dying and necrotic neutrophils are anti-inflammatory secondary to the release of $\alpha$-defensins. JImmunol. 2009;183(3):2122-2132.

117. Serhan CN. Pro-resolving lipid mediators are leads for resolution physiology. Nature. 2014;510(7503):92-101.

118. Serhan CN, Chiang N, Van Dyke TE. Resolving inflammation: dual anti-inflammatory and pro-resolution lipid mediators. Nat Rev Immunol. 2008;8(5):349-361.

119. Serhan CN. Novel lipid mediators and resolution mechanisms in acute inflammation: to resolve or not? Am J Pathol. 2010;177(4):1576-1591.

120. Schwab JM, Chiang N, Arita M, Serhan CN. Resolvin E1 and protectin D1 activate inflammation-resolution programmes. Nature. 2007;447(7146):869-874.

121. Ariel A, et al. Apoptotic neutrophils and T cells sequester chemokines during immune response resolution through modulation of CCR5 expression. Nat Immunol. 2006;7(11):1209-1216.

122. Cocucci E, Racchetti G, Meldolesi J. Shedding microvesicles: artefacts no more. Trends Cell Biol. 2009;19(2):43-51.

123. Camussi G, Deregibus MC, Bruno S, Cantaluppi $\mathrm{V}$, Biancone L. Exosomes/microvesicles as a mechanism of cell-to-cell communication. Kidney Int. 2010;78(9):838-848.

124. Timár CI, et al. Antibacterial effect of microvesicles released from human neutrophilic granulocytes. Blood. 2013;121(3):510-518.

125. Rhys HI, Dell'Accio F, Pitzalis C, Moore A, Norling LV, Perretti M. Neutrophil microvesicles from healthy control and rheumatoid arthritis patients prevent the inflammatory activation of macrophages. EBioMedicine. 2018;29:60-69.

126. Gasser O, Schifferli JA. Activated polymorphonuclear neutrophils disseminate anti-inflammatory microparticles by ectocytosis. Blood . 2004;104(8):2543-2548.

127. Eken C, Gasser O, Zenhaeusern G, Oehri I,
Hess C, Schifferli JA. Polymorphonuclear neutrophil-derived ectosomes interfere with the maturation of monocyte-derived dendritic cells. JImmunol. 2008;180(2):817-824.

128. Headland SE, et al. Neutrophil-derived microvesicles enter cartilage and protect the joint in inflammatory arthritis. Sci Transl Med. 2015;7(315):315ra190.

129. De Santo C, et al. Invariant NKT cells modulate the suppressive activity of IL-10-secreting neutrophils differentiated with serum amyloid A. Nat Immunol. 2010;11(11):1039-1046.

130. Kasten KR, Muenzer JT, Caldwell CC. Neutrophils are significant producers of IL-10 during sepsis. Biochem Biophys Res Commun. 2010;393(1):28-31.

131. Davey MS, et al. Failure to detect production of IL-10 by activated human neutrophils. Nat Immunol. 2011;12(11):1017-1018.

132. Tamassia N, et al. Cutting edge: An inactive chromatin configuration at the IL-10 locus in human neutrophils. JImmunol. 2013;190(5):1921-1925.

133. Perretti M, D'Acquisto F. Annexin A1 and glucocorticoids as effectors of the resolution of inflammation. Nat Rev Immunol. 2009;9 (1):62-70.

134.Perretti M, Dalli J. Exploiting the Annexin A pathway for the development of novel antiinflammatory therapeutics. Br JPharmacol. 2009;158(4):936-946.

135. Scannell M, et al. Annexin-1 and peptide derivatives are released by apoptotic cells and stimulate phagocytosis of apoptotic neutrophils by macrophages. JImmunol. 2007;178(7):4595-4605.

136.Scannell M, Maderna P. Lipoxins and annexin-1: resolution of inflammation and regulation of phagocytosis of apoptotic cells. ScientificWorldJournal. 2006;6:1555-1573.

137. McArthur S, Gobbetti T, Kusters DH, Reutelingsperger CP, Flower RJ, Perretti M. Definition of a novel pathway centered on lysophosphatidic acid to recruit monocytes during the resolution phase of tissue inflammation. JImmunol. 2015;195(3):1139-1151.

138. Mathias JR, Perrin BJ, Liu TX, Kanki J, Look AT, Huttenlocher A. Resolution of inflammation by retrograde chemotaxis of neutrophils in transgenic zebrafish. JLeukoc Biol. 2006;80(6):1281-1288.

139. Buckley CD, et al. Identification of a phenotypically and functionally distinct population of longlived neutrophils in a model of reverse endothelial migration. J Leukoc Biol. 2006;79(2):303-311.

140.Woodfin A, et al. The junctional adhesion molecule JAM-C regulates polarized transendothelia migration of neutrophils in vivo. Nat Immunol. 2011;12(8):761-769.

141. Colom B, et al. Leukotriene B4-neutrophil elastase axis drives neutrophil reverse transendothelial cell migration in vivo. Immunity. 2015;42(6):1075-1086

142.Wu D, et al. Reverse-migrated neutrophils regulated by JAM-C are involved in acute pancreatitis-associated lung injury. Sci Rep. 2016;6:20545.

143. Elks PM, et al. Activation of hypoxia-inducible factor-1 $\alpha$ (Hif-1 $\alpha$ ) delays inflammation resolution by reducing neutrophil apoptosis and reverse migration in a zebrafish inflammation model. Blood. 2011;118(3):712-722.

144.Tharp WG, et al. Neutrophil chemorepulsion in 
defined interleukin-8 gradients in vitro and in vivo. J Leukoc Biol. 2006;79(3):539-554.

145. Blázquez-Prieto J, López-Alonso I, Huidobro C, Albaiceta GM. The emerging role of neutrophils in repair after acute lung injury. Am J Respir Cell Mol Biol. 2018;59(3):289-294.

146.Ambruso DR, et al. Human neutrophil immunodeficiency syndrome is associated with an inhibitory Rac2 mutation. Proc Natl Acad Sci U S A. 2000;97(9):4654-4659.

147. Lakshman R, Finn A. Neutrophil disorders and their management. JClin Pathol. 2001;54(1):7-19. 148. Besner GE, et al. Recombinant human granulocyte colony-stimulating factor promotes wound healing in a patient with congenital neutropenia. J Pediatr Surg. 1992;27(3):288-291.
149. Cody DT, Funk GF, Wagner D, Gidley PW, Graham SM, Hoffman HT. The use of granulocyte colony stimulating factor to promote wound healing in a neutropenic patient after head and neck surgery. Head Neck. 1999;21(2):172-175.

150. Fillmore WJ, Leavitt BD, Arce K. Dental extraction in the neutropenic patient. JOral Maxillofac Surg. 2014;72(12):2386-2393. 